\title{
Selection of efficient controller for flow process in a Conical Tank
}

\author{
A.Nisha ${ }^{1}$, A.Nazreen Banu ${ }^{1}$, M.Shehenaz Fathima ${ }^{1}$, R.Hari Sudhan ${ }^{1}$, S.Ramachandran ${ }^{2}$ \\ Student, Department of Instrumentation and Control Engineering, Saranathan College of Engineering, Trichy ${ }^{1}$ \\ Asst.Professor, Department of Instrumentation and Control Engineering, Saranathan College of Engineering, Trichy ${ }^{2}$
}

\begin{abstract}
This paper deals with the control of level in a conical tank which has nonlinear behaviour and the real time system design are analysed. The implementation in SIMULINK is explained. In many industries, control of non linear process is a challenging task and hence controlling the level in a Conical tank is a complex issue. For each stable operating point, a First Order Process model has been identified using process reaction curve method and the Control is done. The appropriate method is identified by comparing various controllers.
\end{abstract}

Keywords: Conical tank, Non-linear process.

\section{INTRODUCTION}

In many process industries, controlling the level and flow of a tank is so tedious and complexity. Especially in a conical tank, since its area of cross section is changing continuously, it exhibits a non linear characteristics and hence controlling the tank is a tough task. Conical tanks are extensively used in process industries, petrochemical industries, food process industries and wastewater treatment industries and it provides drainages for solid mixtures, slurries and viscous liquids.

The main objective is to implement the suitable controller design for the conical tank system to maintain the desired level by using flow as controlling variable. Many industries may use Proportional Integral Derivative controller (PID) since its tuning is simple. The conical tank is highly unstable and hence various types of controllers are designed and comparison is made between different controllers and most suitable controller for the process is identified.

\section{EXPERIMENTAL SETUP}

The conical tank system is highly non linear system. In this system, flow is used as a controlling variable and level is a controlled variable. Level is sensed by using a level sensor and signal is fed to the signal conditioning circuit. The process is interfaced with personal computer and it acts as the controller where the desired set point is compared with process output. The output from controller is sent to drive circuit which consists of power electronic devices like SCR, TRIAC, DIAC etc. The control action is taken by final control element and thus the desired level of conical tank is maintained.

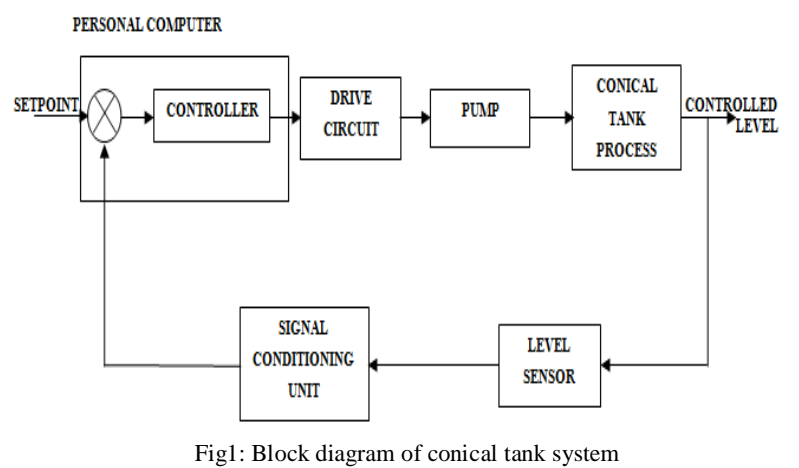

\section{III.MATHEMATICAL MODELLING}

The conical tank process is shown in figure2. By using inflow rate $\mathrm{Q}$, the level of a tank $\mathrm{H}$ is maintained at steady state.

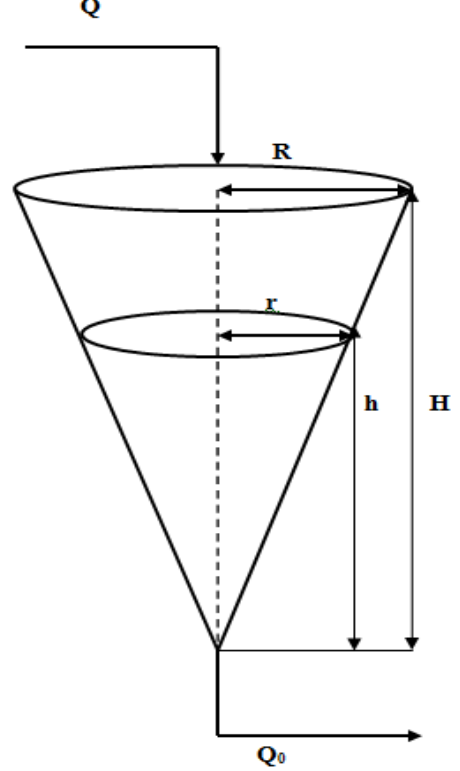

Fig2. Conical tank

Q -Flow rate of the inlet stream

So -Flow rate of the outlet stream

$\mathrm{R}$-Maximum radius of the conical tank

$r$-Radius of the conical tank at steady state

$\mathrm{H}$ - Maximum height of the conical tank

$\mathrm{h}$-Height of the conical tank at steady state

The area of the conical tank is given by

$$
\begin{gathered}
A=\pi r^{2} \\
\tan \theta=\frac{\mathrm{r}}{\mathrm{h}}=\frac{\mathrm{R}}{\mathrm{H}} \\
r=R * \frac{h}{H}
\end{gathered}
$$

According to Law of conservation of mass,

Inflow rate - Outflow rate $=$ Rate of change of Accumulation 


\section{IV.CONTROLLER TUNING}

\section{A. PID CONTROLLER}

In case of unusual characteristics of the plant, the characteristics can be changed by adding a compensator in the control system to obtain the desired output. The most common and simplest controller is a PID controller. The reason PID controllers are so popular is that using PID provides the designer a larger number of options and those options mean that there are more possibilities for changing the dynamics of the system in a way that helps the designer to control the respective process station. The PID controller reads the sensor and then computes the desired actuator value required to compensate the error by calculating the values of proportional gain, integral gain, derivative gain and summing these three values to produce output.

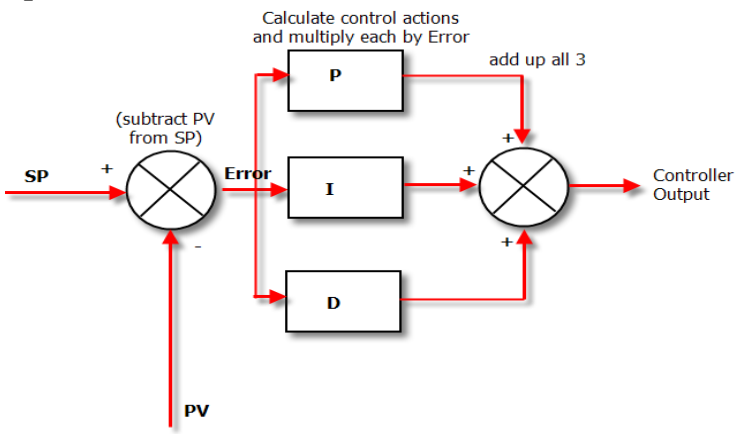

Fig3. Block diagram of PID controller

The PID controller is designed by adjusting the parameters $\mathrm{kp}$,ki and $\mathrm{kd}$ values.

$\mathrm{u}(t)=\mathrm{MV}(t)=K_{p} e(t)+K_{i} \int_{0}^{t} e(\tau) d \tau+K_{d} \frac{d}{d t} e(t)$

e: Error $=S P-P V^{\prime}$

$t$ : Time or instantaneous time (the present)

$\tau$ : Variable of integration; takes on values from time 0 to the present $t$.

$\mathrm{K}_{\mathrm{p}}$ - Proportional gain

$\mathrm{K}_{\mathrm{i}}$-Integral gain

$\mathrm{K}_{\mathrm{d}}$-derivative gain

B. MODEL PREDICTIVE CONTROL

In recent days apart from traditional PID controller Model Predictive Controller is preferred in most of the industrial applications because it has several advantages. Model predictive control (MPC) refers to a class of computer control algorithms that utilize an explicit process model to predict the future response of a given plant. At each control interval an MPC algorithm attempts to optimize future plant behavior by computing a sequence of future manipulated variable adjustments. The first input in the optimal sequence is then sent into the plant, and the entire calculation is repeated at subsequent control intervals. The basic MPC controller can be designed with proper restrictions on the prediction horizon and model length. The prediction horizon has to be kept sufficiently larger than control horizon. The optimization cost function is given by:

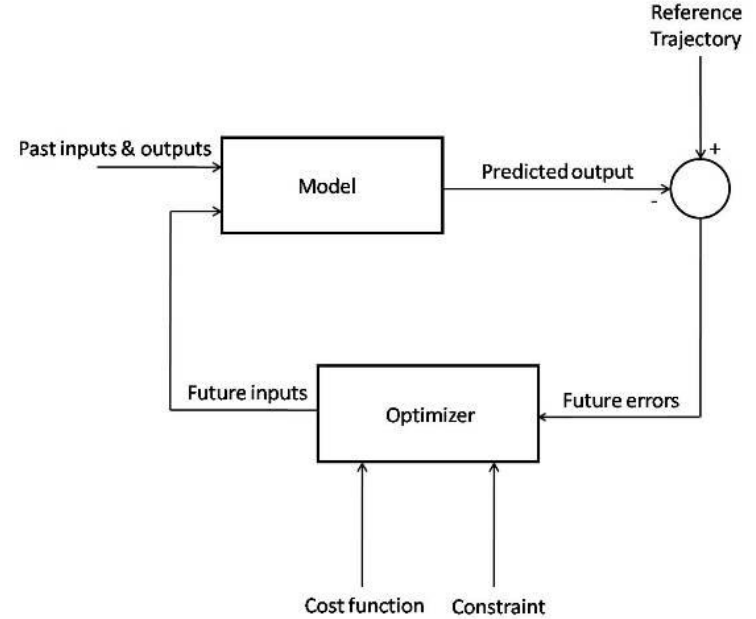

Fig 4 Block diagram of MPC controller

C.INTERNAL MODEL CONTROL

Internal Model Control (IMC) is a commonly used technique that provides a transparent mode for the design and tuning of various types of control. In this report, we analyze that the various concepts of IMC design and IMC based PID controller has been designed for a plant transfer function to incorporate the advantages of PID controller in IMC. The IMC-PID controller does have a good set-point tracking but poor disturbance response mainly for the process which has a minimum time-delay/time-constant ratio. But, for many process control applications, rejection of disturbance for the unstable processes is more important than set point tracking.

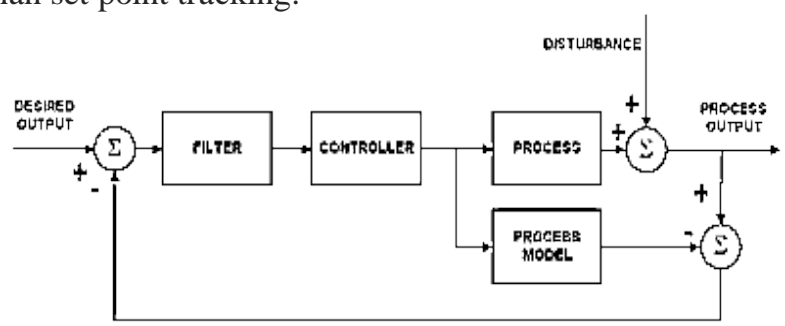

Fig 5 Block diagram of IMC controller

TABLE 1

TUNING FORMULA

\begin{tabular}{|c|c|c|c|c|c|}
\hline $\begin{array}{c}\text { CONTROLL } \\
\text { ER }\end{array}$ & K.Kc & $\tau_{\mathrm{I}}$ & $\tau_{\mathbf{D}}$ & $\tau_{\mathrm{F}}$ & $\begin{array}{c}\text { RECOMMEN } \\
\text { DED } \\
\lambda / \mathrm{d}(\lambda>0.2 \tau \\
\text { always })\end{array}$ \\
\hline PID & $\frac{2 \tau+d}{2(\lambda+d}$ & $\tau+\frac{d}{2}$ & $\frac{\lambda d}{2 \tau+d}$ & $\frac{\lambda d}{2(\lambda+}$ & $>0.25$ \\
\hline PI & $\frac{\tau}{\lambda}$ & $\tau$ & - & - & $>1.7$ \\
\hline $\begin{array}{c}\text { IMPROVED } \\
\text { PI }\end{array}$ & $\frac{2 \tau+d}{2 \lambda}$ & $+\frac{d}{2}$ & - & - & $>1.7$ \\
\hline
\end{tabular}

\section{COMPARISION AND RESULT}

The graph has been plotted for time Vs controlled output for PID, IMC, and MPC controller. It is observed that three controllers reaches the steady state at different time. 


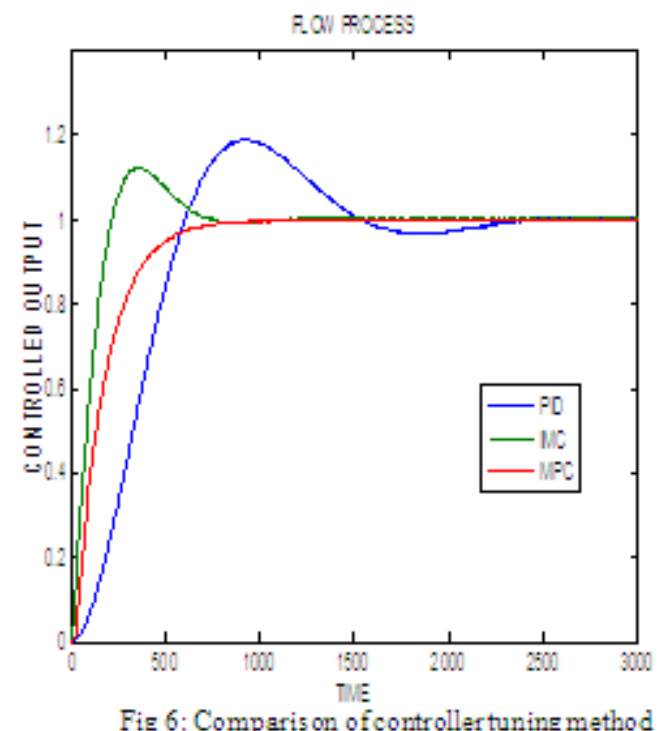

\section{VI.CONCLUSION}

The controllers are tuned for various methods by using their respective formulas, and therefore they are plotted against tuning methods. Based on the simulation results, the first comparison is made between PID and IMC and it is observed that IMC gives better result. Second comparison is made between IMC and MPC and it has been found that MPC shows best result. Hence MPC is found to be the suitable controller for Non- linear flow process.

\section{REFERENCES}

[1] Abhishek Sharma and Nithya Venkatesan (2013) 'Comparing PI controller Performance for Non Linear Process Model', proceedings of International Journal of Engineering Trends and Technology, Vol.4, No.3, pp. 242-245.

[2] Marshiana, D. and Thirusakthimurugan, P. (2012) 'Design of Ziegler Nichols Tuning controller for a Non-linear System', proceedings of International Conference on Computing and Control Engineering, pp.121-124.

[3] Warier, S.R., Venkatesh, S. (2012) 'Design of controllers based on MPC for a conical tank system', proceedings of Advances in Engineering, Science and Management, pp. 309-313.

[4] Mohammad Shahrokhi and AlirezaZomorrodi, Comparison of PID Controller Tuning Methods.

[5] D.AngelineVijula, K.Vivetha, K.Gandhimathi,T.Praveena (2014), Model based Controller Design for Conical Tank System.InternationalJournalofComputerApplications(0975 8887) Volume85 - No12.

[6] S.Abirami, H.Kala, P.B.Nevetha, B.Pradeepa, R.Kiruthiga and P.Sujithra (2014), "Performance Comparison of Different Controllers for Flow Process", International Journal of ComputerApplications90 (19):17-21.

[7]. P.Aravind1, M.Valluvan2, S.Ranganathan (2013), Modelling andSimulation of Non Linear Tank,International Journal of AdvancedResearch in Electrical, Electronics and Instrumentation Engineering, Vol. 2, Issue 2 